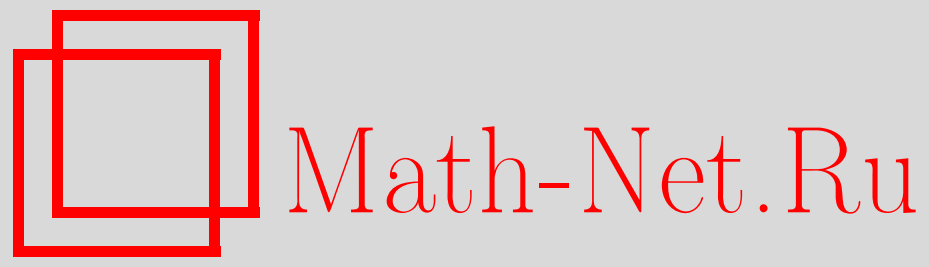

С. В. Нагаев, Нижние оценки для вероятностей больших уклонений сумм независимых случайных величин, Теория вероятн. и ее примен., 2001, том 46, выпуск 1, 50-73

DOI: https://doi.org/10.4213/tvp3951

Использование Общероссийского математического портала MathNet.Ru подразумевает, что вы прочитали и согласны с пользовательским соглашением

http://www . mathnet.ru/rus/agreement

Параметры загрузки:

IP : 54.209 .52 .79

26 апреля 2023 г., 09:20:28

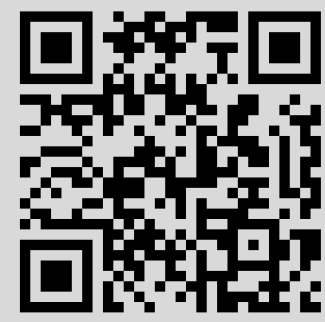




\title{
НИЖНИЕ ОЦЕНКИ ДЛЯ ВЕРОЯТНОСТЕЙ БОЛЬШИХ УКЛОНЕНИЙ СУММ НЕЗАВИСИМЫХ СЛУЧАЙНЫХ ВЕЛИЧИН ${ }^{1)}$
}

\begin{abstract}
Выводятся нижние оценки вероятностей больших уклонений для суммы независимых случайных величин. Область, в которой действуют эти оценки, описывается в терминах ляпуновского отношения. Полученные оценки сравниваются с нижними оценками, принадлежащими Колмогорову, Феллеру, Ленарту и Архангельскому.
\end{abstract}

Ключевые слова и фразы: большие уклонения, метод сопряженных распределений, независимые случайные величины, неравенство Колмогорова, ляпуновское отношение, оценки Берри-Эссеена, свертка функций распределения, условие Бернштейна, характеристическая функция.

1. Введение. Формулировка и обсуждение результатов. Пусть $X_{1}, X_{2}, \ldots, X_{n}$ - независимые случайные величины с нулевыми средними и конечными третьими моментами, $S=\sum X_{j}$. Здесь и в дальнейшем $\sum$ означает $\sum_{1}^{n}$, случай $n=\infty$ не исключается.

Мы будем заниматься нижними оценками вероятности $\mathbf{P}\{S>x\}$. Обозначим $\sigma_{j}^{2}=\mathbf{E} X_{j}^{2}, B^{2}=\sum \sigma_{j}^{2}, \beta_{j}=\mathbf{E}\left|X_{j}\right|^{3}, C=\sum \beta_{j}$. Пусть $L=C / B^{3}$ - ляпуновское отношение.

Пусть $\Phi(x)$ - стандартный нормальный закон. Обозначим $\Phi_{1}(x)$ функцию Миллса $\sqrt{2 \pi}(1-\Phi(x)) e^{x^{2} / 2}$ (по поводу функции Миллса см., например, $\left[1\right.$, с. 204-205]). Положим $\Psi(x)=x \Phi_{1}(x)$.

Первая нижняя оценка вероятности $\mathbf{P}\{S>x\}$ была получена А. Н. Колмогоровым [2] для равномерно ограниченных случайных величин $\left|X_{j}\right|<M$ в связи с доказательством закона повторного логарифма. В наших обозначениях неравенство Колмогорова выглядит следуюшим образом: если $x^{2}>512$ u $a=x M / B \leqslant \frac{1}{256}$, mo

$$
\mathbf{P}\{S>B x\}>\exp \left\{-\frac{x^{2}}{2}(1+\varepsilon)\right\},
$$

${ }^{*}$ Институт математики им. С.Л. Соболева, Уншерситетский пр., 4, 630090 Новосибирск, Россия; e-mail: nagaev-s@mail.ru

1) Работа выполнена при финансовой поддержке Российского фонда фундаментальных исследований (код проекта 96-01-01529). 
где $\varepsilon=\max \left\{64 \sqrt{a}, 32 \sqrt{\ln x^{2}} / x\right\}$. Сформулированный ниже результат принадлежит Ленарту [4].

Eсли $\left|X_{k}\right|<M u 0<x M / B<\frac{1}{12}$, mo

$$
\mathbf{P}\{S>B x\}=\exp \left\{-\frac{x^{2}}{2} Q(x)\right\}\left(1-\Phi(x)+\tau \frac{M}{B} e^{-x^{2} / 2}\right),
$$

где $Q(x)=\sum_{1}^{\infty} q_{k} x^{k}, q_{1}<M / 3 B, q_{k}<8^{-1}(12 M / B)^{k}, k=2,3, \ldots, \infty$, $|\tau|<7,465$ (см. также по этому поводу [5, с. 308]). Этот результат по форме совпадает с результатом Феллера [3] и отличается от последнего несколько меньшими значениями постоянных.

Из (1) нетрудно извлечь неравенство

$$
\mathbf{P}\{S>B x\} \geqslant(1-\Phi(x)) \exp \left\{-c(\gamma) \frac{M x^{3}}{B}\right\}\left(1-7,465 \sqrt{2 \pi} \frac{M x}{B \Psi(x)}\right),
$$

где $1<x<\gamma B / M, c(\gamma)=\frac{1}{6}+9 \gamma /(1-12 \gamma), \gamma<\frac{1}{12}$.

Неравенство (2) действует в более широкой области, нежели неравенство Колмогорова, и, кроме того, оно значительно точнее. Чтобы показать последнее, нам понадобятся некоторые вычисления. Прежде всего, используя тождество (76) (см. п. 2), имеем при условии $x^{2}>512$

$$
1-\Phi(x)>\left(1-\frac{1}{x^{2}}\right) \frac{e^{-x^{2} / 2}}{\sqrt{2 \pi} x}>\frac{e^{-x^{2} / 2-\ln c x}}{\sqrt{2 \pi}}
$$

где $c=\frac{512}{511}$. Далее, при $x^{2}>512$ справедливо неравенство $\sqrt{\ln x}<$ $0,0781 x$, т.е. $\sqrt{2} \ln x<0,0781 x \sqrt{\ln x^{2}}$. При $x^{2}>512$ и $c=\frac{512}{511}$

$$
\ln c x=\left(1+\frac{\ln c}{\ln x}\right) \ln x<1,00063 \ln x .
$$

В результате мы приходим к оценке

$$
\ln c x<0,111 \frac{x^{2}}{2} \frac{\sqrt{\ln x^{2}}}{x} .
$$

С другой стороны,

$$
0,111 \frac{\sqrt{\ln x^{2}}}{x}<0,00346 \varepsilon,
$$

где $\varepsilon$ имеет тот же смысл, что и в неравенстве Колмогорова.

Комбинируя (3) и (5), мы заключаем, что

$$
1-\Phi(x)>\frac{\exp \left\{-\left(x^{2} / 2\right)(1+0,00346 \varepsilon)\right\}}{\sqrt{2 \pi}}
$$

если $x^{2}>512$. Оценим теперь $1-7,465 \sqrt{2 \pi} M x /(B \Psi(1))$ в предположении, что $x^{2}>512$ и $a:=x M / B \leqslant \frac{1}{256}$. Заметим, прежде всего, что

$$
\frac{7,465}{\Psi(1)}<11,397 \text {. }
$$


Следовательно,

$$
z:=7,465 \sqrt{2 \pi} \frac{M x}{B \Psi(1)}<0,113 .
$$

Используя теперь неравенство (80) (см. ниже п. 2), имеем

$$
1-z>\exp \left\{-z-\frac{z^{2}}{1-z}\right\}>\exp \{-1,128 z\}>\exp \left\{-32,75 \frac{M x}{B}\right\} \text {. }
$$

Очевидно, $x<x^{3} / 512$, если $x^{2}>512$. Следовательно,

$$
1-z>\exp \left\{-0,024 \frac{M x^{3}}{B}\right\} \text {. }
$$

Условие $a \leqslant \frac{1}{256}$ соответствует условию $\gamma=\frac{1}{256}$ в неравенстве (2). Несложные вычисления показывают, что

$$
c\left(\frac{1}{256}\right)<0,204 \text {. }
$$

Комбинируя неравенства (8), (9) и $a \leqslant \sqrt{a} / 16$, мы получаем

$$
\begin{aligned}
(1-z) \exp \left\{-c\left(\frac{1}{256}\right) \frac{M x^{3}}{B}\right\} & >\exp \left\{-0,268 \frac{M x^{3}}{B}\right\} \\
& \geqslant \exp \left\{-0,0335\left(\frac{x^{2} \sqrt{a}}{2}\right)\right\} .
\end{aligned}
$$

Заметим, что

$$
0,0335 \sqrt{a} \leqslant 53 \cdot 10^{-5} \varepsilon
$$

Из (2), (6), (10), (11) следует оценка

$$
\mathbf{P}\{S>B x\}>\frac{\exp \left\{-\left(1+4 \cdot 10^{-3} \varepsilon\right) x^{2} / 2\right\}}{\sqrt{2 \pi}} .
$$

Из приведенных выкладок видно, насколько трудоемким является сравнение уже имеющихся оценок, не говоря уже о выводе новых.

Сравним теперь оценку (2) с оценкой из недавней работы [6]:

$$
\mathbf{P}\{S>B x\}>\exp \left\{-(1+50 \alpha) \frac{x^{2}}{2}\right\}
$$

которая справедлива при условии

$$
\frac{1}{\alpha}<x<\frac{\alpha M}{B}, \quad 0<\alpha<10^{-2} .
$$

Прежде всего, вследствие (76)

$$
1-\Phi(x)>\frac{\exp \left\{\ln \left(1-1 / x^{2}\right)-\ln x-x^{2} / 2\right\}}{\sqrt{2 \pi}} .
$$

Далее, $1-1 / x^{2}>0,9999, \ln x<0,0461 x<0,0461 \alpha x^{2}$. Следовательно,

$$
1-\Phi(x)>0,9999 \frac{\exp \left\{-(1+0,0922 \alpha) x^{2} / 2\right\}}{\sqrt{2 \pi}} \text {. }
$$


Несложные вычисления с использованием (7), (80) и условия (13) приводят к оценке

$$
1-7,465 \sqrt{2 \pi} \frac{M x}{B \Psi(1)}>\exp \left\{-28,89 \frac{M x}{B}\right\}>\exp \left\{-58 \cdot 10^{-4} \alpha \frac{x^{2}}{2}\right\} \text {. }
$$

Из (2), (14), (15) следует, что при условии (13)

$$
\mathbf{P}\{S>B x\}>\frac{\exp \left\{-(1+0,098 \alpha) x^{2} / 2\right\}}{\sqrt{2 \pi}} .
$$

Таким образом, оценка (2) точнее, чем (12).

До сих пор речь шла о нижних оценках в случае, когда слагаемые равномерно ограничены. Перейдем к случаю, когда это условие не выполняется. При условии Бернштейна

$$
\mathbf{E}\left|X_{j}\right|^{k} \leqslant \frac{k !}{2} \sigma_{j}^{2} c^{k-2}, \quad j=1, \ldots, n, k=3,4, \ldots,
$$

нижнюю оценку можно извлечь из работы [7] (см. по этому поводу [8]). Существенное продвижение было сделано в работе А.Н. Архангельского [8], который заменил условие равномерной ограниченности слагаемых условием $\sup _{j} \mathbf{E}\left|X_{j}\right|^{2+\delta} / \sigma_{j}^{2}<\infty, \delta>0$.

Наша цель в настоящей работе заключается в том, чтобы заменить индивидуальные ляпуновские отношения на обычное ляпуновское отношение. Более точно, мы хотим получить аналог оценки (2) с заменой $M / B$ на $L$.

Сформулируем наши результаты, полученные в этом направлении.

Теорема 1. Пусть выполнены условия

$$
0<x<(1-4 \gamma)\left(\frac{\gamma}{L} \wedge \frac{\alpha B}{\max _{j} \sigma_{j}}\right), \quad \gamma<\frac{1}{16}, e \alpha^{2}<\gamma .
$$

Тогда

$$
\begin{aligned}
\mathbf{P}\{S>B x\}> & (1-\Phi(x)) \exp \left\{-\left(c_{1}(\gamma) L+c_{2}(\gamma, \alpha) \sum \frac{\sigma_{j}^{3}}{B^{3}}\right) x^{3}\right\} \\
& \times\left(1-\frac{\left(c_{3}(\gamma) L+c_{4}(\alpha) \sum \sigma_{j}^{3} / B^{3}\right) x}{\Psi(x)}\right),
\end{aligned}
$$

где $c_{1}(\gamma) \leqslant 1,5 /(1-4 \gamma)^{3}, c_{2}(\gamma, \alpha) \leqslant 2 \alpha /(1-4 \gamma)^{3}, c_{3}(\gamma) \leqslant 9,79+76,26 \gamma$, $c_{4}(\alpha) \leqslant 106 \alpha$.

Близкий результат сформулирован в [9], однако без указания значений постоянных.

Сравним оценку (18) с соответствующим результатом из [8]. Положим $c=\max _{j} \beta_{j} / \sigma_{j}^{2}$. Из теоремы 1 этой работы следует, что при $a:=x c / B<\alpha$ и $x>x_{0}$

$$
\mathbf{P}\{S>B x\}>(1-\Phi(x)) \exp \left\{-\rho a x^{2}\right\}(1-K a),
$$


где $K=K\left(\alpha, x_{0}\right), \rho=\rho(\alpha)$, но явный вид зависимости от $\alpha$ и $x_{0}$ не приводится. Отмечается только, что при $\alpha=0,031, K=16,37, \rho=3,06$. Заметим, что $c^{-1}<\left(B^{2} / C\right) \wedge\left(1 / \max \sigma_{j}\right)$. Поэтому из условия $a<\alpha$ следует, что

$$
x<\alpha\left(\frac{1}{L} \wedge \frac{B}{\max \sigma_{j}}\right) .
$$

Если $\alpha<\frac{3}{4}$, то условие (20) является частным случаем (17). Если $\alpha=0,031$, то (20) переходит в условие (17) с $\gamma=\alpha=0,032$. В этом случае оценка (18) принимает вид

$$
\dot{\mathbf{P}}\{S>B x\}>(1-\Phi(x)) e^{-1,858 L x^{3}}(1-8,25 L x) .
$$

Учитывая, что $L x \leqslant a$, мы видим, что в рассматриваемом случае неравенство (18) дает значительно более точный результат, нежели (19).

Следствие 1. Если выполнены условия (17), то

$$
\mathbf{P}\{S>B x\}>(1-\Phi(x)) \exp \left\{-c_{1}(\alpha, \gamma) L x^{3}\right\}\left(1-\frac{c_{2}(\alpha, \gamma) L x}{\Psi(x)}\right)
$$

гдe

$$
c_{1}(\alpha, \gamma) \leqslant \frac{1,5+2 \alpha}{(1-4 \gamma)^{3}}, \quad c_{2}(\alpha, \gamma) \leqslant 9,79+76,26 \gamma+106 \alpha .
$$

Полагая в (17) $\gamma=\frac{1}{20}, \alpha=\frac{1}{20}$ и учитывая, что $\Psi(1,7)>0,805$, мы получаем

Следствие 2. Eсли $1,7<x<\left((1 / L) \wedge\left(B / \max _{j} \sigma_{j}\right)\right) / 25$, mo

$$
\begin{aligned}
\mathbf{P}\{S>B x\} & >(1-\Phi(x)) e^{-3,13 L x^{3}}(1-23,4 L x) \\
& >0,06(1-\Phi(x)) e^{-3,13 L x^{3}} .
\end{aligned}
$$

Если случайные величины $X_{i}$ одинаково распределены, то

$$
\max _{j} \frac{\sigma_{j}}{B}=\frac{1}{\sqrt{n}} \leqslant \frac{C}{B^{3}}=\frac{\beta}{\sigma^{3} \sqrt{n}},
$$

где $\sigma^{2}$ и $\beta$ - соответственно второй и третий абсолютный моменты величин $X_{i}$.

Если $\left|X_{i}\right|<M, i=1, \ldots, n$, то $C / B^{3}<M / B, \max _{j} \sigma_{j} / B<M / B$. Таким образом, как в случае одинаково распределенных, так и в случае ограниченных слагаемых, можно исключить из условия (17) $B / \max _{j} \sigma_{j}$. Сформулируем теперь получающиеся в результате оценки.

Следствие 3. Если $X_{i}$ одинаково распределены $u$

$$
1,7<x \leqslant \frac{1}{25} \sqrt{n} \frac{\sigma^{3}}{\beta}
$$

mo

$$
\begin{aligned}
\mathbf{P}\{S>B x\}> & (1-\Phi(x)) \exp \left\{-\frac{\left(2,35 \beta / \sigma^{3}+0,2\right) x^{3}}{\sqrt{n}}\right\} \\
& \times\left(1-\frac{\left(16,88 \beta / \sigma^{3}+6,58\right) x}{\sqrt{n}}\right) .
\end{aligned}
$$


Для стандартного нормального закона значения $x \geqslant 3$ следует рассматривать как большие. Заметим в этой связи, что значение $x=3$ удовлетворяет условию (24) для $n \geqslant 64^{2}\left(\beta / \sigma^{3}\right)^{2}$. На статистическом языке это означает, что нам нужно произвести не менее $2^{12}=4096$ наблюдений, чтобы иметь возможность воспользоваться неравенством (25) для $x \geqslant 3$.

Следствие 4. Eсли $\left|X_{j}\right|<M u$

$$
1,7<x \leqslant \frac{1}{25} \frac{B}{M}
$$

mo

$$
\mathbf{P}\{S>B x\}>(1-\Phi(x)) \exp \left\{-2,13 \frac{M x^{3}}{B}\right\}\left(1-23,4 \frac{M x}{B}\right) .
$$

Несложные вычисления показывают, что при $1,7<x<\frac{1}{25} M / B$ неравенство (2) дает более точную оценку, нежели следствие 4:

$$
\mathbf{P}\{S>B x\}>(1-\Phi(x)) \exp \left\{-1,291 \frac{M x^{3}}{B}\right\}\left(1-23,2 \frac{M x}{B}\right) .
$$

Можно исключить $\max \sigma_{j} / B$ из условия (17) и в общем случае, однако ценой значительного увеличения постоянных, а именно, справедлива

Теорема 2. Если $\frac{3}{2}<x<\gamma / L, \gamma<\frac{1}{14^{2}}$, то

$$
\mathbf{P}\{S>B x\}>(1-\Phi(x)) \exp \left\{-c_{1}(\gamma) L x^{3}\right\}\left(1-c_{2}(\gamma) L x\right),
$$

¿de

$$
c_{1}(\gamma) \leqslant \frac{29+38 \sqrt{\gamma}}{(1-28 \gamma)^{3}}+\frac{41}{\sqrt{\gamma}}, \quad c_{2}(\gamma) \leqslant 60+1630 \gamma+1212 \sqrt{\gamma}+\frac{0,63}{\sqrt{\gamma}} .
$$

Следствие 5. Если $\frac{3}{2}<x<\gamma / L, \gamma<\frac{1}{14^{2}}$, то

$$
\mathbf{P}\{S>B x\}>(1-\Phi(x)) \exp \left\{-1419 L x^{3}\right\}(1-164 L x) .
$$

Сформулированные выше нижние оценки лучше использовать в комбинации с неравенством

$$
\mathbf{P}\{S>B x\}>\frac{1}{2} \sum_{j} \mathbf{P}\left\{X_{j}>2 B x\right\}
$$

которое справедливо, по крайней мере, для $x>2$ (см. $[10$, с. 759]). Если распределения случайных величин $X_{j}$ имеют тяжелые хвосты, то предлагаемые нами оценки точнее, чем оценка (30), лишь для сравнительно небольших значений $x$.

Предположим для простоты, что $X_{j}$ одинаково распределены $\mathbf{P}\{X>x\}>c / x^{t}, x>1, t>3$. Если $x>b \sqrt{\ln n}$, где $b>\sigma(t-2)^{1 / 2}$, то для достаточно больших $n$

$$
n \mathbf{P}\{S>2 \sigma x \sqrt{n}\}>2(1-\Phi(x)) .
$$


Поэтому при $x>b \sqrt{\ln n}$ оценка (30) оказывается точнее оценки (25), если $n$ достаточно велико.

Рассмотрим теперь совсем конкретный пример. Пусть плотность $p(x)$ определяется равенством

$$
p(x)= \begin{cases}0, & |x|<1, \\ \frac{2}{|x|^{5}}, & |x| \geqslant 1 .\end{cases}
$$

Сравним оценки (25) и (30) в случае, когда $X_{j}$ одинаково распределены с плотностью $p(x)$, в точке $x=3$. В данном случае $\sigma^{2}=2, \beta=4$.

При $\gamma=\frac{1}{16}$ оценка (25) применима при $n>11250$. Неравенство (25) при $n>11250$ принимает вид

$$
\mathbf{P}\{S>3 \sqrt{2 n}\}>0,13 e^{-1,12}(1-\Phi(3))>0,042(1-\Phi(3))>56 \cdot 10^{-6} .
$$

С другой стороны, пользуясь неравенством (30), мы получаем для $n>11250$ оценку

$$
\mathbf{P}\{S>3 \sqrt{2 n}\}>\frac{n}{2} \mathbf{P}\{X>6 \sqrt{2 n}\}>\frac{1}{3^{4} \cdot 2^{20}} \sim 11 \cdot 10^{-9} .
$$

Мы видим, что при $x=3, n=11250$ оценка (25) на четыре порядка точнее, чем оценка (30). Обратим внимание читателя, что в рассматриваемом примере $x<\sigma((t-2) \ln n)^{1 / 2}$.

Посмотрим, что в данном случае дает оценка Берри-Эссеена

$$
|\Phi(3)-\mathbf{P}\{S>3 \sqrt{2 n}\}|<c_{0} \sqrt{\frac{2}{n}}
$$

с постоянной $c_{0}=0,7655$ [11] (см. также $[12$, с. 229]).

При $n=11250$ мы получаем тривиальную оценку

$$
\mathbf{P}\{S>3 \sqrt{2 n}\}>1-\Phi(3)-0,0103>-0,091 \text {. }
$$

Нетривиальная оценка получается для $n>643 \cdot 10^{3}$.

Наши рассмотрения лишний раз показывают, какую большую роль играют постоянные, когда дело касается практических вычислений. На первый взгляд, постоянная $\frac{3}{64}$ в условии (24) не слишком мала, но тем не менее именно она является причиной того, что неравенство (25) справедливо только при больших $n$.

Доказательство теорем 1 и 2 технически сложно. В основе его лежит модификация метода, предложенного в [13]. Последний является комбинацией урезания с последующим применением метода сопряженных распределений (по поводу последнего см. [5], а также [14]).

В п. 2, в котором собрано 19 лемм, проводится подготовка к доказательству теорем 1 и 2 . В п. 3 доказывается теорема 1 , а в п. $4-$ теорема 2.

В дальнейшем нам понадобятся следующие определения и обозначения. 
Определим срезку $X(y)$ случайной величины $X$ равенством

$$
X(y)= \begin{cases}X, & X \leqslant y \\ 0, & X>y .\end{cases}
$$

Положим $r(h, y)=\mathbf{E} e^{h X(y)}, a(h, y)=\mathbf{E} X(y) e^{h X(y)}, \sigma^{2}(h, y)=$ $\mathbf{E} X^{2}(y) e^{h X(y)}, \beta(h, y)=\mathbf{E}|X(y)|^{3} e^{h X(y)} / r(h, y)$.

\section{Обозначим}

$$
a(y)=a(0, y), \quad \sigma^{2}(y)=\sigma^{2}(0, y), \quad \beta(y)=\beta(0, y)
$$

Пусть

$$
A(y)=\sum a_{j}(y), \quad B^{2}(y)=\sum \sigma^{2}(y)
$$

Положим

$$
\begin{gathered}
m(h, y)=\frac{a(h, y)}{r(h, y)} \equiv \frac{\partial}{\partial h} r(h, y), \quad M(h, y)=\sum m_{j}(h, y), \\
b^{2}(h, y)=\frac{\sigma^{2}(h, y)}{r(h, y)}-m^{2}(h, y) \equiv \frac{\partial^{2}}{\partial h^{2}} \ln r(h, y) .
\end{gathered}
$$

Индекс $j$ здесь и ниже означает, что соответствующая характеристика относится к случайной величине $X_{j}$. Заметим, что $M(h, y)$ как функция $h$ возрастает при $y>0$, поскольку

$$
\frac{\partial}{\partial h} M(h, y)=\sum b_{j}^{2}(h, y)>0 .
$$

$\mathrm{C}$ другой стороны, $M(0, y)=A(y) \leqslant 0$. Поэтому уравнение относительно $h M(h, y)=x$ при $x \geqslant 0$ имеет единственное решение, которое мы обозначим через $h(x, y)$.

Положим $F(x)=\mathbf{P}\{X<x\}, B^{2}(h, y)=\sum_{j} b_{j}^{2}(h, y)$. Пусть

$$
C(h, y)=\sum \mathbf{E} \frac{e^{h X_{j}(y)}}{r_{j}(h, y)}\left|X_{j}(y)-m_{j}(h, y)\right|^{3}
$$

\section{2. Вспомогательные результаты.}

Лемма 1. Если $0<h \leqslant 1 / y$, mо

$$
0<r(h, y)-1-a(y) h<\frac{e h^{2} \sigma^{2}(y)}{2} .
$$

Д оказ а тельст в о. Утверждение леммы следует из тождества

$$
r(h, y)=1+a(y) h+\int_{-\infty}^{y}\left(e^{h u}-1-h u\right) d F(u)
$$

и неравенства $0<e^{u}-1-u<e u^{2} / 2, u<1$.

Положим $\beta_{+}=\mathbf{E}\left\{X^{3} ; X>0\right\}, \beta_{-}=\mathbf{E}\left\{|X|^{3} ; X<0\right\}$.

Лемма 2. Если $0<h \leqslant 1 / y$, то

$$
\begin{gathered}
a(h, y) \geqslant a(y), \\
-\frac{\beta_{-} h^{2}}{2}<a(h, y)-a(y)-\sigma^{2}(y) h<\beta_{+}\left(\frac{(e-2)}{y^{2}} \wedge \frac{e h^{2}}{2}\right) .
\end{gathered}
$$


Д ок а з а т е л с т в о. Неравенство (32) следует из тождества

$$
a(h, y)-a(y)=\int_{-\infty}^{y}\left(e^{h u}-1\right) u d F(u)
$$

и неравенства $\left(e^{h u}-1\right) u \geqslant 0$. Выведем теперь неравенство (33). Очевидно, $a(h, y)-a(y)-\sigma^{2}(y) h=\int_{-\infty}^{y}\left(e^{h u}-1-h u\right) u d F(u)$. Остается воспользоваться неравенствами

$$
\begin{aligned}
0<e^{h u}-1-h u & <\left(\frac{e h^{2}}{2} \wedge \frac{e-2}{y^{2}}\right) u^{2}, \quad u>0, \\
e^{h u}-1-h u & <\frac{h^{2} u^{2}}{2}, \quad u<0 .
\end{aligned}
$$

Лемма 3. Пусть $0<h<1 / y$,

$$
y>\left(\alpha^{-1} \max _{j} \sigma_{j}\right) \vee \frac{C}{\gamma B^{2}}, \quad e \alpha^{2}<\gamma .
$$

Тогда при $\gamma<1$

$$
M(h, y)>\left((1-2 \gamma) h-\frac{2 \gamma}{y}\right) B^{2} .
$$

Д ок а з а т е л в с т в о. Учитывая, что $a(y) \leqslant 0, a(h, y)-a(y) \geqslant 0$ (см. лемму 2), имеем

$$
M(h, y)>\frac{A(y)}{\min _{j} r_{j}(h, y)}+\frac{\sum_{j}\left(a_{j}(h, y)-a_{j}(y)\right)}{\max _{j} r_{j}(h, y)} .
$$

В силу леммы 1 при условии (35)

$$
1-e^{-1} \gamma<1-\frac{\sigma^{2}}{y^{2}}<r(h, y)<1+\frac{e \sigma^{2}}{2 y^{2}}<1+\frac{e \alpha^{2}}{2} .
$$

Далее,

$$
B^{2}(y)>B^{2}-\frac{C}{y}>(1-\gamma) B^{2} .
$$

Используя (39) и левое неравенство в (33), мы получаем

$$
\sum_{j}\left(a_{j}(h, y)-a_{j}(y)\right)>B^{2}(y) h-\frac{C h^{2}}{2}>\left(1-\frac{3 \gamma}{2}\right) B^{2} h .
$$

Наконец,

$$
|A(y)|<\frac{C}{y^{2}}<\frac{\gamma B^{2}}{y} .
$$

Комбинируя (37)-(41), мы заключаем, что

$$
M(h, y)>\frac{(1-3 \gamma / 2) B^{2} h}{1+e \alpha^{2} / 2}-\frac{\gamma B^{2}}{\left(1-e^{-1} \gamma\right) y} .
$$

Заметим, что при $e \alpha^{2}<\gamma$

$$
\frac{1}{1+e \alpha^{2} / 2}>1-\frac{e \alpha^{2}}{2}>1-\frac{\gamma}{2}>\frac{1}{2} .
$$

Из двух последних оценок следует утверждение леммы. 
Лемма 4. Пусть выполнены условия (35). Тогда при $\gamma \leqslant \frac{1}{4}$

$$
h(x, y)<\frac{x+2 \gamma B^{2} / y}{(1-2 \gamma) B^{2}} \leqslant \frac{1}{y}
$$

ecлu

$$
x \leqslant \frac{(1-4 \gamma) B^{2}}{y} \text {. }
$$

Д ок аз ат т льст в о. Обозначим $M_{1}(h, y)=((1-2 \gamma) h-$ $2 \gamma / y) B^{2}$. Согласно (36), при $0<h<1 / y$

$$
M(h, y)>M_{1}(h, y) .
$$

Пусть $h_{1}(x, y)$ - корень уравнения $M_{1}(h, y)=x$. Очевидно,

$$
M_{1}\left(\frac{1}{y}, y\right)=\frac{(1-4 \gamma) B^{2}}{y}
$$

Вследствие (45) и (46) при $x<(1-4 \gamma) B^{2} / y$

$$
h(x, y)<h_{1}(x, y)=\frac{x+2 \gamma^{2} B^{2} / y}{(1-2 \gamma) B^{2}} \leqslant \frac{1}{y}
$$

что и требовалось доказать.

Положим

$$
\begin{gathered}
Q(h, y)=\sum\left(r_{j}(h, y)-1\right), \\
C^{+}=\sum \mathbf{E}\left\{X_{j}^{3} ; X_{j}>0\right\}, \quad C^{-}=\sum \mathbf{E}\left\{\left|X_{j}\right|^{3} ; X_{j}<0\right\} .
\end{gathered}
$$

Лемма 5. Если $h<1 / y$, mo

$$
A(y) h+\frac{B^{2}(y) h^{2}}{2}-\frac{C^{-} h^{3}}{6}<Q(h, y)<\frac{e B^{2} h^{2}}{2} .
$$

Д ок азат е л с т в о. Нетрудно видеть, что

$$
\begin{aligned}
r(h, y) & =1+a(y) h+\frac{\sigma^{2}(y) h^{2}}{2}+\int_{-\infty}^{y}\left(e^{h u}-1-h u-\frac{h^{2} u^{2}}{2}\right) d F(u) \\
& >1+a(y) h+\frac{\sigma^{2}(y) h^{2}}{2}-\frac{h^{3} \beta_{-}}{6} .
\end{aligned}
$$

Отсюда вытекает левое неравенство в (47). Правое неравенство следует из оценки $r(h, y)-1<e h^{2} \sigma^{2} / 2$.

Лемма 6. Пусть $0<\gamma<\frac{1}{16}$,

$$
\begin{gathered}
y=\frac{(1-4 \gamma) B^{2}}{x}, \\
x<(1-4 \gamma) B^{2}\left(\frac{\gamma B^{2}}{C} \wedge \frac{\alpha}{\max _{j} \sigma_{j}}\right), \\
e \alpha^{2}<\gamma .
\end{gathered}
$$


Tогда прu $h=h(x, y)$

$$
Q(h, y)-h x>-\frac{x^{2}}{2 B^{2}}-1,5 C\left(\frac{x}{(1-4 \gamma) B^{2}}\right)^{3} .
$$

Д ок аз а т л в ст во. Заметим, прежде всего, что в силу (48)-(50) у удовлетворяет условию (35). Таким образом, выполня.ются условия леммы 4, т.е. имеет место неравенство (43). Из (43) и (48) следует

$$
h(x, y)<\frac{x}{(1-4 \gamma) B^{2}}=\frac{1}{y} .
$$

Комбинируя (47), (52), мы заключаем, что

$$
Q(h, y)>\frac{B^{2} h^{2}}{2}-C^{+}\left(\frac{h}{y^{2}}+\frac{h^{2}}{2 y}\right)-C^{-} \frac{h^{3}}{6}>\frac{B^{2} h^{2}}{2}-1,5 \frac{C}{y^{3}} .
$$

Заметим, что $B^{2} h^{2} / 2-h x>-x^{2} /\left(2 B^{2}\right)$ при любом $h>0$. Из двух последних неравенств вытекает утверждение леммы.

Лемма 7. Пусть $0<h<1 / y$ и выполнено условие (35). Тогда npu $\alpha^{2}<1$

$$
h(x, y)>\frac{\left(1-\alpha^{2}\right) x}{(1+e \gamma / 2) B^{2}} .
$$

Д о к а з а т е л в с т в о. Используя правое неравенство (33), имеем $M(h, y) \leqslant\left(B^{2} h+C e h^{2} / 2\right) / \min _{j} r_{j}(h, y)$. Заметим, что в силу (35) $C h^{2}<\gamma B^{2} h$. Согласно левому неравенству (38), при условии (35)

$$
r(h, y)>1-\frac{\sigma^{2}}{y^{2}}>1-\alpha^{2} .
$$

Таким образом,

$$
M(h, y) \leqslant \frac{h(1+e \gamma / 2) B^{2}}{1-\alpha^{2}} .
$$

Отсюда следует утверждение леммы.

Лемма 8. Если выполнены условия (35) $u$ (48), то при $h<1 / y$

$$
\frac{1}{y} \leqslant \frac{1+e \gamma / 2}{(1-4 \gamma)\left(1-\alpha^{2}\right)} h(x, y) .
$$

Д ок а з а т е в с т в о. Утверждение леммы следует из (48) и (53).

Лемма 9. Если $0<h \leqslant 1 / y$, mo

$$
-\frac{\sigma^{2}}{y}<a(h, y)<\sigma^{2}\left(e h \wedge \frac{(e-1)}{y}\right) .
$$

Д ок аз а т е л с т в о. Используя (34), нетрудно показать, что

$$
a(h, y)-a(y)<\sigma^{2}\left(e h \wedge \frac{e-1}{y}\right) .
$$

Остается заметить, что $-\sigma^{2} / y \leqslant a(y) \leqslant 0$, и воспользоваться оценкөй (32). 
Лемма 10. Пусть $0<h \leqslant 1 / y$ и выполнены условия

$$
y>\frac{\max _{j} \sigma_{j}}{\alpha}, \quad \alpha^{2}<\frac{1}{16 e} .
$$

Тогда

$$
\max _{j} \frac{\left|m_{j}(h, y)\right|}{\sigma_{j}}<1,76 \alpha .
$$

Д ок аз а те льс т в о. В силу (54), (56), (57) $|m(h, y)|<$ $(e-1) \alpha \sigma /\left(1-\alpha^{2}\right)$. Остается воспользоваться условием $\alpha^{2}<1 / 16 e$.

Лемма 11. В условиях леммы 10

$$
C(h, y)<2,782 C+19 \alpha \sum \sigma_{j}^{3}
$$

если $\gamma<\frac{1}{16}$.

Д ок а а т е л с с т о. В качестве отправной точки мы используем неравенство

$$
\begin{aligned}
\left(1-\alpha^{2}\right) C(h, y)< & \sum \beta_{j}(h, y)+3 \sum \sigma_{j}^{2}(h, y)\left|m_{j}(h, y)\right| \\
& +3 \sum \alpha_{j}(h, y) m_{j}^{2}(h, y)+\sum\left|m_{j}(h, y)\right|^{3},
\end{aligned}
$$

которое нетрудно получить с помощью (54). Здесь $\alpha(h, y)=$ $\int_{-\infty}^{y}|u| e^{h u} d F(u)$.

Вследствие оценок (58) и

$$
\sigma^{2}(h, y)<e \sigma^{2}
$$

имеем

$$
\sum \sigma_{j}^{2}(h, y)\left|m_{j}(h, y)\right|<4,79 \alpha \sum \sigma_{j}^{3} .
$$

Аналогично,

$$
\begin{gathered}
\sum \beta_{j}(h, y)<e C, \quad \sum \alpha_{j}(h, y) m_{j}^{2}(h, y)<8,42 \alpha^{2} \sum \sigma_{j}^{3}, \\
\sum\left|m_{j}(h, y)\right|^{3}<5,46 \alpha^{3} \sum \sigma_{j}^{3} .
\end{gathered}
$$

Из $(60),(62),(63)$ следует желаемый результат.

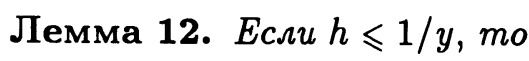

$$
(e-1) \frac{\beta}{y}>\sigma^{2}(h, y)-\sigma^{2}(y)>-\beta h .
$$

Д ок а з а т е ль с т в о. Очевидно, $\sigma^{2}(h, y)=\sigma^{2}(y)+\int_{-\infty}^{y} u^{2}\left(e^{h u}-\right.$ 1) $d F(u)$. Далее,

$$
\frac{e-1}{y} \beta_{+}>\int_{0}^{y} u^{2}\left(e^{h u}-1\right) d F(u)>0, \quad \int_{u<0} u^{2}\left(1-e^{h u}\right) d F(u)<h \beta_{-},
$$

откуда и следует утверждение леммы. Тогда

Лемма 13. Пусть $0<h \leqslant 1 /$ и и выполнены условия (35) с $\gamma<\frac{1}{16}$.

$$
B^{2}(h, y)>\frac{32}{33}\left(1-2 \gamma-3,2 \alpha^{2}\right) B^{2} .
$$


Д ок аз а т е л ь с т в о. Мы начнем с оценки $\sum \sigma_{j}^{2}(h, y)>B^{2}(y)-$ $\sum \beta_{j} / y>B^{2}-2 C / y>(1-2 \gamma) B^{2}$, которая следует из (35) и (64).

Принимая теперь во внимание (38), мы находим, что

$$
\frac{\sum \sigma_{j}^{2}(h, y)}{r_{j}(h, y)}>\frac{32}{33}(1-2 \gamma) B^{2} .
$$

Согласно (58), $\sum m_{j}^{2}(h, y)<3,1 \alpha^{2} B^{2}$. Комбинируя приведенные выше неравенства, мы получаем желаемый результат.

Лемма 14. Пусть $0<h<1 /$ и и выполнены условия (35) с $\gamma<\frac{1}{16}$. Tогдa

$$
\left|h B(h, y)-\frac{M(h, y)}{B(y)}\right|<\frac{2,78 \alpha \sum \sigma_{j}^{3} h}{B y}+1,686 \frac{C}{B y^{2}} \text {. }
$$

Д ок аз а т е л в с т в о. Прежде всего, согласно (33), (35) и (54),

$$
m(h, y)=\sigma^{2}(y) h+\frac{\theta}{1-\alpha^{2}}\left(\beta_{+}(e-2) y^{-2}+\alpha h y^{-1} \sigma^{2}(y) \sigma\right) .
$$

Здесь и ниже $\theta$ означает величину, по модулю не превосходящую 1 . Отсюда

$$
\frac{M(h, y)}{B(y)}=h B(y)+1,023 \theta\left(\frac{(e-2) C}{y^{2} B(y)}+\frac{\alpha h \sum \sigma_{j}^{3}}{y B(y)}\right) .
$$

Аналогично в силу (64) и (54)

$$
\frac{\sum \sigma_{j}^{2}(h, y)}{r_{j}(h, y)}=\sum \sigma_{j}^{2}(y)+1,023 \theta\left(\frac{(e-1) C}{y}+\frac{\alpha \sum \sigma_{j}^{3}}{y}\right) .
$$

Далее, согласно (57) и (56),

$$
\sum m_{j}^{2}(h, y)=\theta\left(\frac{e-1}{1-\alpha^{2}}\right)^{2} \alpha \sum \frac{\sigma_{j}^{3}}{y} .
$$

Следовательно,

$$
\begin{aligned}
B^{2}(h, y) & :=\frac{\sum \sigma_{j}^{2}(h, y)}{r_{j}(h, y)}-m_{j}^{2}(h, y) \\
& =B^{2}(y)+\theta\left(1,023 \frac{(e-1) C}{y}+3,1 \alpha \sum \frac{\sigma_{j}^{3}}{y}\right)
\end{aligned}
$$

Очевидно,

$$
h B(h, y)=h B(y)+h(B(h, y)-B(y))
$$

Далее,

$$
B(h, y)-B(y)=\frac{B^{2}(h, y)-B^{2}(y)}{B(h, y)+B(y)} .
$$

Заметим, что $B(h, y)+B(y)>2 \sqrt{B(y) B(h, y)}$. Используя лемму 13 , имеем при $\gamma \leqslant \frac{1}{16}: 1 / \sqrt{B(h, y)}<1,065 / \sqrt{B}$. Аналогично, учитывая (39), 
получаем $1 / \sqrt{B(y)}<1,017 / \sqrt{B}$. Из трех последних оценок следует

$$
\frac{1}{B(h, y)+B(y)}<\frac{0,541}{B} \text {. }
$$

Комбинируя (69), (71), (72), имеем

$$
B(h, y)-B(y)=\frac{\theta\left(0,951 C+1,68 \alpha \sum \sigma_{j}^{3}\right)}{B y} .
$$

Из (67), (70), (73) получаем, учитывая (39), оценку (66).

Лемма 15. Для любых $0<u_{1} \leqslant u_{2}$

$$
1<\frac{\Phi_{1}\left(u_{1}\right)}{\Phi_{1}\left(u_{2}\right)}<\exp \left\{\frac{u_{2}-u_{1}}{u_{1}^{2} \Phi_{1}\left(u_{1}\right)}\right\} .
$$

Д ок а з а т е л в с т в о. Исследуем функцию $\ln \Phi_{1}(u)$. Очевидно,

$$
\frac{d}{d u} \ln \Phi_{1}(u)=u-\frac{e^{-u^{2} / 2}}{\sqrt{2 \pi}(1-\Phi(u))}=u\left(1-\frac{1}{\sqrt{2 \pi} u e^{u^{2} / 2}(1-\Phi(u))}\right) .
$$

Нетрудно видеть, что

$$
\sqrt{2 \pi} u e^{u^{2} / 2}(1-\Phi(u))=1-u e^{u^{2} / 2} \int_{u}^{\infty} e^{-v^{2} / 2} v^{-2} d v .
$$

Функция $g(u)=u e^{u^{2} / 2}(1-\Phi(u))$ возрастает. Действительно,

$$
\frac{d}{d u} \ln g(u)=u+\frac{1}{u}-\frac{e^{-u^{2} / 2}}{\sqrt{2 \pi}(1-\Phi(u))} .
$$

Далее, в силу (76)

$$
1-\Phi(u)=\frac{e^{-u^{2} / 2}}{u \sqrt{2 \pi}}-\frac{1}{\sqrt{2 \pi}} \int_{u}^{\infty} e^{-v^{2} / 2} v^{-2} d v
$$

Отсюда

$$
\frac{e^{-u^{2} / 2}}{\sqrt{2 \pi}}-u(1-\Phi(u))=\frac{u}{\sqrt{2 \pi}} \int_{u}^{\infty} e^{-v^{2} / 2} v^{-2} d v<u^{-1}(1-\Phi(u)) .
$$

Таким образом,

$$
\left(u+\frac{1}{u}\right)(1-\Phi(u))>\frac{e^{-u^{2} / 2}}{\sqrt{2 \pi}},
$$

т.е. $\frac{d}{d u} \ln g(u) \geqslant 0$. Следовательно, $g(u)$ возрастает при $u>0$. Это в силу (76) означает, что функция $\Phi_{2}(u)=u e^{u^{2} / 2} \int_{u}^{\infty} v^{-2} e^{-v^{2} / 2} d v$ убывает. Согласно (76),

$$
\Phi_{2}(u)=1-\sqrt{2 \pi} u e^{u^{2} / 2}(1-\Phi(u))=1-\Psi(u) .
$$

Используя равенство $1 /(1-x)=1+x /(1-x)$, мы заключаем, что при $u \geqslant u_{1}$

$$
1<\frac{1}{1-\Phi_{2}(u)}<1+\frac{\Phi_{2}(u)}{1-\Phi_{2}\left(u_{1}\right)} .
$$


Из (75)-(78) следует

$$
0<-\frac{d}{d u} \ln \Phi_{1}(u)<\frac{u \Phi_{2}(u)}{1-\Phi_{2}\left(u_{1}\right)}, \quad u>u_{1} .
$$

Нетрудно видеть, что $\Phi_{2}(u)<1 / u^{2}$. Таким образом,

$$
0<-\frac{d}{d u} \ln \Phi_{1}(u)<\frac{1}{\left(1-\Phi_{2}\left(u_{1}\right)\right) u}
$$

а это равносильно утверждению леммы.

Лемма 16. Для любых $0<u_{1}<u_{2}$

$$
\frac{1-\Phi\left(u_{2}\right)}{1-\Phi\left(u_{1}\right)}>\exp \left\{\frac{\left(u_{1}-u_{2}\right) u_{2}}{\Psi\left(u_{1}\right)}\right\} \text {. }
$$

Д о к а з а т е л в с т в о. Рассмотрим функцию $g(x)=\ln (1-\Phi(x))$. Очевидно, $g^{\prime}(x)=-\Phi^{\prime}(x) /(1-\Phi(x))=-x / \Psi(x)$. Функция $\Psi(x)$ возрастает (см. доказательство леммы 15). Поэтому для $u_{2}>x>u_{1}$

$$
-g^{\prime}(x)<\frac{u_{2}}{\Psi_{1}\left(u_{1}\right)} .
$$

Из этой оценки легко следует утверждение леммы.

Положим $\lambda(x)=(1-a x) e^{-c x^{3}}(1-\Phi(x))$, где $a$ и $c$ - некоторые постоянные.

Лемма 17. Если $а>0$, то функиия $\lambda(x)$ выпукла при $0<x \leqslant 1 / a$.

Д о к а з а т е л ь с т в о. Очевидно, $\lambda(x)=e^{g(x)}$, где $g(x)=\ln (1-$ $a x)+\ln (1-\Phi(x))-c x^{3}$. Нетрудно видеть, что

$$
\begin{aligned}
& g^{\prime}(x)=-3 c x^{2}-\frac{a}{1-a x}-\frac{1}{\Phi_{1}(x)}, \\
& g^{\prime \prime}(x)=-6 c x-\frac{a^{2}}{(1-a x)^{2}}-\frac{1}{\Phi_{1}^{2}(x)}+\frac{x}{\Phi_{1}(x)} .
\end{aligned}
$$

Если $0<x \leqslant 1 / a$, то $g^{\prime 2}(x)>6 c x+a^{2}(1-a x)^{-2}+1 / \Phi_{1}^{2}(x)$. Таким образом, при $0 \leqslant x \leqslant 1 / a$ имеем $g^{\prime 2}(x)+g^{\prime \prime}(x)>0$ и, следовательно, $\lambda^{\prime \prime}(x)=\left(g^{\prime 2}(x)+g^{\prime \prime}(x)\right) e^{g(x)} \geqslant 0$, что и требовалось доказать.

Лемма 18. Для любого $0<x<1$

$$
e^{-x-x^{2} /(1-x)}<1-x
$$

Д ок аз а т е ль с т в о. Нетрудно видеть, что $1 /(1+y)>e^{-y}$, т.е.

$$
e^{-y}<1-\frac{y}{1+y}
$$

Полагая $x=y /(1+y)$, имеем $y=x /(1-x)=x+x^{2} /(1-x)$. Подставляя это выражение в (81), мы получаем нужный результат. 
Лемма 19. Если выполнено условие $\max _{j} \sigma_{j}<B \varepsilon$, то для любого $h>0$

$$
\begin{aligned}
\mid h \int_{0}^{\infty} & e^{-h x}\left(\mathbf{P}\{S<x\}-\Phi\left(\frac{x}{B}\right)\right) d x \mid<\frac{L(\varepsilon)}{6 \sqrt{2 \pi}\left(1-0,61 L^{2 / 3}(\varepsilon)\right)^{3 / 2}} \\
& +\frac{2,473 C^{2} h}{\pi B^{5}}+\frac{L^{2 / 3}}{\pi}\left(0,607 e^{-0,82 L^{-2 / 3}}+0,304 e^{-1,64 L^{-2 / 3}}\right) \\
& +L^{2} e^{-0,225 L^{-2}}
\end{aligned}
$$

где $L(\varepsilon)=C / B_{\varepsilon}^{3}, B_{\varepsilon}^{2}=\left(1-\varepsilon^{2}\right) B^{2}$.

Д ок а з а т е л с т в о. Прежде всего,

$$
\begin{aligned}
& \int_{0}^{\infty} e^{-h x}\left(\Phi\left(\frac{x}{B}\right)-\mathbf{P}\{S<x\}\right) d x=\frac{1}{2 \pi} \int_{-\infty}^{\infty} \frac{g(t)-e^{-B^{2} t^{2} / 2}}{i t(h+i t)} d t \\
&=\frac{1}{2 \pi}\left(\int_{|t|<\tau_{1}} \frac{g(t)-e^{-B^{2} t^{2} / 2}}{i t(h+i t)} d t+\int_{\tau_{1}<|t| \leqslant \tau_{2}} \frac{g(t) d t}{i t(h+i t)}\right. \\
& \quad+\int_{\tau_{2}<|t|<\tau_{3}} \frac{g(t) d t}{i t(h+i t)}+\int_{|t|>\tau_{3}} \frac{g(t) d t}{i t(h+i t)} \\
&\left.-\int_{|t|>\tau_{1}} \frac{e^{-B^{2} t^{2} / 2}}{i t(h+i t)} d t\right)=\sum_{1}^{5} I_{j} .
\end{aligned}
$$

Здесь $g(t)=\mathbf{E} e^{i t S}, \tau_{1}=(6 / C)^{1 / 3}, \tau_{2}=1,5 B^{2} / C, \tau_{3}=2,4 B^{2} / C$. Для любой характеристической функции $f(t)$

Отсюда

$$
|f(t)|<\exp \left\{\frac{1}{2}\left(|f(t)|^{2}-1\right)\right\} .
$$

$$
\left|\mathbf{E} e^{i t X}\right|<\exp \left\{-\frac{\sigma^{2} t^{2}}{2}+\frac{\beta|t|^{3}}{6}\right\}
$$

поскольку $|f(t)|^{2}<1-\sigma^{2} t^{2}+\beta|t|^{3} / 3$. Используя теперь неравенство $\left|\prod_{1}^{n} a_{j}-\prod_{1}^{n} b_{j}\right|<\sum_{j=1}^{n}\left|a_{j}-b_{j}\right| \prod_{k=1}^{j-1}\left|a_{k}\right| \prod_{l=j+1}^{n}\left|b_{l}\right|$, имеем

$$
\left|g(t)-e^{-B^{2} t^{2} / 2}\right|<\frac{C|t|^{3}}{6} \exp \left\{-\frac{B_{\varepsilon}^{2} t^{2}}{2}+\frac{C|t|^{3}}{6}\right\} .
$$

Несложные вычисления показывают, что при $|t|<\tau_{1}$

$$
\frac{B_{\varepsilon}^{2} t^{2}}{2}-\frac{C|t|^{3}}{6}>\frac{B_{\varepsilon}^{2}}{2}\left(1-0,61 L^{2 / 3}(\varepsilon)\right) \text {. }
$$

Из (85) и (86) следует оценка

$$
\left|I_{1}\right|<\frac{L(\varepsilon)}{6 \sqrt{2 \pi}\left(1-0,61 L^{2 / 3}(\varepsilon)\right)^{3 / 2} h} .
$$

В силу (84)

$\pi h\left|I_{2}\right|<\int_{\tau_{1}<t<\tau_{2}} \exp \left\{-\frac{B^{2} t^{2}}{2}+\frac{C t^{3}}{6}\right\} \frac{d t}{t}<\int_{t>\tau_{1}} e^{-B^{2} t^{2} / 4} \frac{d t}{t}<\frac{2 e^{-B^{2} \tau_{1}^{2} / 4}}{B^{2} \tau_{1}^{2}}$ 
Отсюда

$$
\left|I_{2}\right|<\frac{2}{6^{2 / 3} \pi h} L^{2 / 3} e^{-6^{2 / 3} / 4 L^{-2 / 3}}<\frac{0,607}{\pi h} L^{2 / 3} e^{-0,82 L^{-2 / 3}} .
$$

Аналогично,

$$
\left|I_{3}\right|<\frac{L^{2}}{\pi h} e^{-0,225 L^{-2}}, \quad\left|I_{4}\right|<\frac{0,304}{\pi h} L^{2 / 3} e^{-1,64 L^{-2 / 3}} .
$$

Используя (84), получаем оценку

$$
\int_{t>\tau_{2}} \frac{|g(t)| d t}{t^{2}}<\sum_{k=0}^{\infty} \frac{1}{s_{k}^{2}} \int_{\Delta_{k}}|g(t)| d t
$$

где $s_{k}=\tau_{2}+1,3 k B^{2} / C, \Delta_{k}=\left[s_{k}, s_{k+1}\right)$. В силу леммы 3 из работы [15]

$$
\int_{\Delta_{k}}|g(t)| d t<\frac{5,86}{B} \text {. }
$$

С другой стороны,

$$
\sum_{0}^{\infty} \frac{1}{s_{k}^{2}}<0,422 \frac{C^{2}}{B^{4}}
$$

Из (90)-(92) следует

$$
\left|I_{5}\right|<2,473 \frac{C^{2}}{\pi B^{5}}
$$

Комбинируя тождество (83) и оценки (87)-(89), (93), мы получаем желаемый результат.

3. Доказательство теоремы 1. Поскольку $X_{j}(y) \leqslant X_{j}$, то

$$
\mathbf{P}\{S>x\} \geqslant \mathbf{P}\{S(y)>x\}
$$

где $S(y)=\sum X_{j}(y)$. Пусть $G(x ; y)=\mathbf{P}\{S(y)<x\}$. Нетрудно видеть, что

$$
\mathbf{P}\{S(y)>x\}=R(h, y) \int_{x}^{\infty} e^{-h u} G_{h}(d u),
$$

где $R(h, y)=\mathbf{E} e^{h S(y)}=\prod r_{j}(h, y), G_{h}(d u)=e^{h u} G(d u ; y) / R(h, y)$. Полагая $\bar{G}_{h}(u)=G_{h}(u+x)$, имеем

$$
\mathbf{P}\{S(y)>x\}=R(h, y) e^{-h x} \int_{0}^{\infty} e^{-h u} d \bar{G}_{h}(u) .
$$

Функция распределения $G_{h}$ является сверткой функций распределения $F_{j}(u ; h, y), j=1,2, \ldots, n$, где $F(d u ; h, y)=e^{h u} F(d u ; y) / r(h, y)$. Нетрудно видеть, что $\int_{-\infty}^{y} u F(d u ; y)=m(h, y), \int_{-\infty}^{y}(u-m(h, y))^{2} F(d u ; y)=$ $\sigma^{2}(h, y)$. Следовательно, функции распределения $G_{h}$ соответствуют математическое ожидание $M(h, y)=\sum m_{j}(h, y)$ и дисперсия $B^{2}(h, y)=$ $\sum b_{j}^{2}(h, y)$.

В дальнейшем будем считать, что $y$ удовлетворяет условию (48). Положим теперь $h=h(x, y)$. Тогда, согласно определению, $x=M(h, y)$ (см. п. 1). 
Не нарушая общности, можно считать, что

$$
x \geqslant 1,7 \text {. }
$$

Действительно, согласно оценке Берри-Эссеена,

$$
\mathbf{P}\{S>x\}>(1-\Phi(x))\left(1-\frac{c_{0} L}{1-\Phi(x)}\right),
$$

где $c_{0}<0,7915$ (см. [11], а также [12, с. 229]). С другой стороны, $c_{3}(\gamma)$ в (18) не может быть меньше 9,79. Поэтому оценка (97) заведомо точнее (18), если $(1 / \sqrt{2 \pi}) e^{-x^{2} / 2}>c_{0} / 9,79$, т.е. $x<1,7$.

\section{Рассмотрим тождество}

$$
\begin{aligned}
\int_{0}^{\infty} e^{-h u} d \bar{G}_{h}(u) & =\int_{0}^{\infty} e^{-h u} d \Phi\left(\frac{u}{B(h, y)}\right)+h \int_{0}^{\infty} e^{-h u} r_{h}(u) d u-r_{h}(0) \\
& =I_{1}+h I_{2}-r_{h}(0)
\end{aligned}
$$

где $r_{h}(u)=\bar{G}_{h}(u)-\Phi(u / B(h, y))$. Нетрудно показать, что

$$
I_{1}=\frac{1}{\sqrt{2 \pi}} e^{h^{2} B^{2}(h, y) / 2} \int_{h B(h, y)}^{\infty} e^{-u^{2} / 2} d u=\frac{\Phi_{1}(h B(h, y))}{\sqrt{2 \pi}} .
$$

Пусть $\Delta(h, y)=h B(h, y)-M(h, y) / B(y)$. Предположим, что $\Delta(h, y)>0$. В силу леммы 14 , а также равенства (48)

$$
\begin{aligned}
\frac{\Delta(h, y) B(y)}{x} & <\frac{x\left(1,686 C+2,78 \alpha \sum \sigma_{j}^{3}\right)}{(1-4 \gamma)^{2} B^{4}} \\
& <\frac{x\left((1,686+20 \gamma) C+6,26 \alpha \sum \sigma_{j}^{3}\right)}{B^{4}} .
\end{aligned}
$$

Полагая в лемме $15 u_{1}=x / B(y), u_{2}=h B(h, y)$ и используя предыдушую оценку, имеем

$$
\begin{aligned}
\sqrt{2 \pi} I_{1}= & \Phi_{1}(h B(h, y))>\Phi_{1}\left(\frac{x}{B(y)}\right) \\
& \times \exp \left\{-\frac{\left((1,686+20 \gamma) C+6,26 \alpha \sum \sigma_{j}^{3}\right) x}{B^{4} \Psi(x / B)}\right\} .
\end{aligned}
$$

Мы учитывали здесь, что $\Psi(x / B)<\Psi(x / B(y))$. Пусть теперь $u_{1}=x / B$, $u_{2}=x / B(y)$. Подставляя эти значения в неравенство (74), находим, что

$$
\Phi_{1}\left(\frac{x}{B(y)}\right)>\Phi_{1}\left(\frac{x}{B}\right) \exp \left\{-\frac{1-B / B(y)}{\Psi(x / B)}\right\} .
$$

Используя (39) и (48), имеем

$$
\frac{B}{B(y)}-1=\frac{B^{2}-B^{2}(y)}{B(y)(B+B(y))}<\frac{C}{2 y B^{2}(y)}<\frac{C x}{2(1-\gamma)(1-4 \gamma) B^{4}}
$$

Таким образом,

$$
\Phi_{1}\left(\frac{x}{B(y)}\right)>\Phi_{1}\left(\frac{x}{B}\right)\left(1-\frac{(1+6,76 \gamma) C x}{2 \Psi(x / B) B^{4}}\right)
$$


Из (100) и (101) следует

$$
\sqrt{2 \pi} I_{1}>\Phi_{1}\left(\frac{x}{B}\right) \exp \left\{-\frac{\left((2,186+23,38 \gamma) C+6,26 \alpha \sum \sigma_{j}^{3}\right) x}{B^{4} \Psi(x / B)}\right\} .
$$

Если $\Delta(h, y)<0$, то $\Phi_{1}(h B(h, y))>\Phi_{1}(x / B(y))$. Отсюда, используя (101), мы получаем, что при $\Delta(h, y)<0$

$$
\sqrt{2 \pi} I_{1}>\Phi_{1}\left(\frac{x}{B}\right)\left(1-\frac{(1+6,76 \gamma) C x}{2 \Psi(x / B) B^{4}}\right) .
$$

Перейдем теперь к оценке $I_{2}$. Для этого мы применим лемму 19 к сумме независимых случайных величин с функцией распределения $\bar{G}_{h}$. Hетрудно видеть, что ляпуновское отношение $L(h, y)$, соответствующее $\bar{G}_{h}$, равно $C(h, y) / B^{3}(h, y)$. Из леммы 11 следует, что при $\alpha^{2}<1 / 16 e$

$$
C(h, y)<5,67 C \text {. }
$$

Положим $B_{1}^{2}(h, y)=\inf _{j}\left(B^{2}(h, y)-b_{j}^{2}(h, y)\right)$. В силу леммы 13 $B_{1}^{2}(h, y)>0,777\left(B^{2}-\sup _{j} \sigma_{j}^{2}\right)$. Заметим, что вследствие (17) $x / B<$ $3 \alpha\left(B /\left(4 \max \sigma_{j}\right)\right)$. Отсюда в силу (96)

$$
\left(\frac{\max \sigma_{j}}{B}\right)^{2}<\left(\frac{3}{4 x}\right)^{2} \alpha^{2}<0,005 \text {. }
$$

Таким образом,

$$
B_{1}^{2}(h, y)>0,773 B^{2} .
$$

Сравнивая (104) и (106), имеем

$$
\frac{C^{2 / 3}(h, y)}{B_{1}^{2}(h, y)}<4,15 L^{2 / 3}
$$

Вследствие (17) и (96)

$$
L<\frac{3}{64 x}<0,028 \text {. }
$$

Комбинируя (107) и (108), получаем после несложных вычислений

$$
R_{1}:=\frac{L_{1}(h, y)}{6 \sqrt{2 \pi}\left(1-0,61 L_{1}^{2 / 3}(h, y)\right)^{3 / 2}}<0,075 L_{1}(h, y),
$$

где $L_{1}(h, y)=C(h, y) / B_{1}^{3}(h, y)$.

В силу (65) и (105)

$$
B_{1}^{2}(h, y)>0,96 B^{2}\left(1-2 \gamma-3,2 \alpha^{2}\right) .
$$

Нетрудно видеть, что

$$
\frac{1}{\left(1-2 \gamma-3,2 \alpha^{2}\right)^{3 / 2}}<\frac{1}{(1-3,178 \gamma)^{3 / 2}}<1+6,3 \gamma<1,394
$$

Из (59), (110), (111) следует

$$
L_{1}(h, y)<\frac{(2,96+18,7 \gamma) C+28,2 \alpha \sum \sigma_{j}^{3}}{B^{3}} .
$$


Сравнивая (109) и (112), получаем

$$
R_{1}<\frac{(0,24+1,515 \gamma) C+2,285 \alpha \sum \sigma_{j}^{3}}{B^{3}} .
$$

В силу (17), (43) и (48) $h(x, y)<x /(1-4 \gamma) B^{2}<\gamma B^{2} / C<B^{2} / 16 C$. Отсюда, учитывая (59) и (65), заключаем, что

$$
\frac{h C^{2}(h, y)}{B^{5}(h, y)}<1,08 \frac{2,782^{2} C+\left(38 \cdot 2,782 \alpha+361 \alpha^{2}\right) \sum \sigma_{j}^{3}}{16(1-3,178 \gamma)^{5 / 2} B^{3}} .
$$

Следовательно,

$$
\frac{2,473 h C^{2}(h, y)}{\pi B^{5}(h, y)}<\frac{(0,412+4,89 \gamma) C+14,91 \alpha \sum \sigma_{j}^{3}}{B^{3}} .
$$

Подставляем теперь в правую часть (82) вместо $L$ и $L(\varepsilon)$ соответственно $L(h, y)$ и $L_{1}(h, y)$. Первые два слагаемых оцениваются посредством (113) и (114). Что касается остальных, то, как показывают вычисления, ими можно пренебречь. В результате мы приходим к оценке

$$
h\left|I_{2}\right|<\frac{(0,652+6,1 \gamma) C+17,195 \alpha \sum \sigma_{j}^{3}}{B^{3}} .
$$

Далее, согласно оценке Берри-Эссеена,

$$
\sup _{u}\left|r_{h}(u)\right|<\frac{c_{0} C(h, y)}{B^{3}(h, y)} .
$$

Применяя теперь леммы 11 и 13 , получаем

$$
\begin{aligned}
\left|r_{h}(0)\right| & <\frac{1,08 c_{0}\left(2,782 C+19 \alpha \sum \sigma_{j}^{3}\right)}{\left(1-2 \gamma-3,5 \alpha^{2}\right)^{3 / 2} B^{3}} \\
& <\frac{(2,38+15 \gamma) C+22,65 \alpha \sum \sigma_{j}^{3}}{B^{3}} .
\end{aligned}
$$

Между прочим, можно было бы воспользоваться неравенством (116) и для оценки $h I_{2}$, поскольку $h\left|I_{2}\right|<\sup _{u}|r(u)|$.

Так до сих пор и поступали, начиная с основополагающей работы Крамера [14]. Однако при этом мы бы проиграли в точности. Чтобы в этом убедиться; достаточно сравнить оценки (115) и (117). В значительной степени потеря точности связана с тем, что оценка для $c_{0}$, которой мы пользовались, не является оптимальной.

Продолжая доказательство, заметим, что в силу (38) и (54) при $e \alpha^{2}<\gamma, \gamma<\frac{1}{16}$

$$
|r(h, y)-1|<\frac{1}{32} \text {. }
$$

Отсюда, используя лемму 18 и неравенство $1+x>e^{x-x^{2} /(1-x)}$, где $0<x<\frac{1}{2}$, имеем

$$
R(h, y)>\exp \left\{Q(h, y)-\frac{32}{31} \sum\left(r_{j}(h, y)-1\right)^{2}\right\}
$$


(определение $Q(h, y)$ см. перед леммой 5). Вследствие (31) и (55) $|r(h, y)-1|<e h^{2} \sigma^{2} / 2 \vee \sigma^{2} h / y \leqslant e \sigma^{2} /\left(2 y^{2}\right)$. Отсюда, используя (31) и условия (48), (49), имеем

$$
\begin{aligned}
\sum\left(r_{j}(h, y)-1\right)^{2} & <\left(\frac{e}{2}\right)^{2} y^{-4} \max _{l} \sigma_{l} \sum \sigma_{j}^{3}<\frac{x^{4} \max _{l} \sigma_{l} \sum \sigma_{j}^{3}}{(1-4 \gamma)^{4} B^{8}}\left(\frac{e}{2}\right)^{2} \\
& <\frac{1,85 \alpha x^{3} \sum \sigma_{j}^{3}}{(1-4 \gamma)^{3} B^{6}}
\end{aligned}
$$

Из (51), (118), (119) следует

$$
e^{-h x} R(h, y)>\exp \left\{-\frac{x^{2}}{2 B^{2}}-\frac{\left(1,5 C+1,91 \alpha \sum \sigma_{j}^{3}\right) x^{3}}{(1-4 \gamma)^{3} B^{6}}\right\} .
$$

Комбинируя (94), (95), (98), (102), (103), (115), (117), (120), получаем

$$
\begin{aligned}
\mathbf{P}\{S>x\}> & {\left[\left(1-\Phi\left(\frac{x}{B}\right)\right) \exp \left\{-\frac{\left((2,186+23,38 \gamma) C+6,26 \alpha \sum \sigma_{j}^{3}\right) x}{B^{4} \Psi(x / B)}\right\}\right.} \\
& \left.-\frac{e^{-x^{2} / 2 B^{2}}}{B^{3}}\left((3,032+21,1 \gamma) C+39,845 \alpha \sum \sigma_{j}^{3}\right)\right] \\
& \times \exp \left\{-\frac{\left(1,5 C+1,98 \alpha \sum \sigma_{j}^{3}\right) x^{3}}{(1-4 \gamma)^{3} B^{6}}\right\} .
\end{aligned}
$$

Очевидно,

$$
e^{-x^{2} / 2 B^{2}}=\sqrt{2 \pi} \frac{(1-\Phi(x / B)) x}{\Psi(x / B) B} .
$$

Из (121) и (122) следует утверждение теоремы.

4. Доказательство теоремы 2. Пусть числа $\gamma$ и $\alpha>0$ связаны равенством $\gamma=\alpha^{2}$. Предположим, что выполняется неравенство

$$
x<\frac{\gamma B^{4}}{C} .
$$

Определим

$$
N(x)=\left\{j: \sigma_{j}<\frac{\alpha B^{2}}{3 x}\right\} .
$$

Положим $S_{1}=\Sigma^{\prime} X_{j}, S_{2}=\Sigma^{\prime \prime} X_{j}$. Здесь и ниже $\Sigma^{\prime}=\sum_{j \in N(x)}, \Sigma^{\prime \prime}=$ $\sum_{j \notin N(x)}$. Пусть $B_{1}^{2}=\sum^{\prime} \sigma_{j}^{2}, B_{2}^{2}=\sum^{\prime \prime} \sigma_{j}^{2}, C_{1}=\sum^{\prime} \beta_{j}$. Тогда

$$
B_{2}^{2}<\frac{3 x C}{\alpha B^{2}}<3 \frac{\gamma B^{2}}{\alpha}=2 \sqrt{\gamma} B^{2} .
$$

Если $\gamma<\frac{1}{14^{2}}$, то $B_{2}^{2}<3 B^{2} / 14$, т.е.

$$
B_{1}^{2}>\frac{11}{14} B^{2} .
$$


Положим $\gamma_{1}=3,5 \gamma$. Очевидно,

$$
\frac{\left(1-4 \gamma_{1}\right) \alpha B_{1}^{2}}{\max _{j \in N(x)} \sigma_{j}} \geqslant \frac{33(1-14 \gamma) x}{14}>2 x,
$$

если $\gamma \leqslant 1 / 14^{2}$. Далее, при $\gamma<\frac{1}{14^{2}}$

$$
\frac{\gamma B^{4}}{C}<\left(\frac{14}{11}\right)^{2} \frac{\gamma_{1} B_{1}^{4}}{3,5 C_{1}}<\frac{\left(1-4 \gamma_{1}\right) \gamma_{1} B_{1}^{4}}{2 C_{1}} .
$$

Таким образом, при $\gamma<\frac{1}{14^{2}}\left(\gamma_{1}<\frac{1}{16}\right)$ выполняется условие

$$
2 x<\left(1-4 \gamma_{1}\right) B_{1}^{2}\left(\frac{\gamma_{1} B_{1}^{2}}{C_{1}} \wedge \frac{\alpha}{\max _{j \in N(x)} \sigma_{j}}\right),
$$

т.е. для $S_{1}$ и $x$, удовлетворяющих неравенству (123), выполняется условие (17) с $\gamma=\gamma_{1}$.

Применяя теперь к $S_{1}$ следствие 1 , мы заключаем, что при $B_{1}<x<$ $\omega=\left(1-4 \gamma_{1}\right) B_{1}^{2}\left(\gamma_{1} B_{1}^{2} / C_{1} \wedge \alpha / \max _{j \in N(x)} \sigma_{j}\right)$

$$
\begin{aligned}
\mathbf{P}\left\{S_{1}>x\right\}> & \left(1-\Phi\left(\frac{x}{B_{1}}\right)\right) \exp \left\{-\frac{c_{1}\left(\alpha, \gamma_{1}\right) C_{1} x^{3}}{B_{1}^{3}}\right\} \\
& \times\left(1-\frac{c_{2}\left(\alpha, \gamma_{1}\right) C_{1} x}{B_{1}^{4} \Psi(1)}\right) \equiv f(x),
\end{aligned}
$$

где $c_{j}(\cdot, \cdot)$ определяются равенствами (22). Отсюда, обозначая $\mathbf{P}\left\{S_{i} \leqslant\right.$ $x\}=G_{i}(x), i=1,2$, имеем

$$
\begin{aligned}
\mathbf{P}\{S>x\} & =\int_{-\infty}^{\infty}\left(G_{1}(x-u)\right) d G_{2}(u) \\
& >\int_{u_{1}}^{u_{2}} f(x-u) d G_{2}(u)+f(x)\left(1-G_{2}\left(u_{2}\right)\right),
\end{aligned}
$$

где $u_{1}=x-\omega, u_{2}=x-B_{1}$. Согласно лемме 17 , функция $f(x-u)$ выпукла по $u$ при $u_{1} \leqslant u<x$. Следовательно, по неравенству Юнга

$$
\int_{u_{1}}^{u_{2}} f(x-u) d G_{2}(u)>p f(x-q),
$$

где $p=G_{2}\left(u_{2}\right)-G_{2}\left(u_{1}\right), q=\int_{u_{1}}^{u_{2}} u d G_{2}(u) / p$. Если $3 B / 2<x<\omega / 2$, то $\left|u_{1}\right|>\omega / 2>3 B / 2$ и, согласно (124),

$$
G_{2}\left(u_{1}\right)<\frac{B_{2}^{2}}{u_{1}^{2}}<\frac{8}{9} \frac{x C}{\alpha B^{4}} .
$$

Оценим теперь величину $q$. Если $x>B$, то в силу (124)

$$
-\int_{u_{1}}^{u_{2}} u d G_{2}(u)<\frac{B_{2}^{2}}{u_{2}}<\frac{3 B_{2}^{2}}{x}<\frac{6 C}{\sqrt{\gamma} B^{2}} .
$$

Далее, $p>1-B_{2}^{2} / u_{1}^{2} \wedge u_{2}^{2}$. Согласно (124), при $3 B / 2<x<\omega / 2$

$$
\frac{B_{2}^{2}}{u_{1}^{2}}<\frac{4}{9} \sqrt{\gamma}, \quad \frac{B_{2}^{2}}{u_{2}^{2}}<\frac{4 B_{2}^{2}}{B^{2}}<4 \sqrt{\gamma} .
$$


Таким образом,

$$
p>1-4 \sqrt{\gamma}>0,465,
$$

если $3 B / 2<x<\omega / 2, \gamma<\frac{1}{56}$. Из (129) и (130) следует

$$
-q<\frac{12,91 C}{\sqrt{\gamma} B^{2}}=\eta
$$

Используя лемму 16 и оценку (131), заключаем, что при $3 B / 2<x<$ $\omega / 2, \gamma<\frac{1}{14^{2}}$

$$
\begin{aligned}
f(x-q)> & \left(1-\Phi\left(\frac{x}{B_{1}}\right)\right) \\
& \times \exp \left\{-\frac{1}{\Psi(3 / 2)}\left(\frac{x \eta}{B_{1}^{2}}+\frac{\eta^{2}}{B_{1}^{2}}\right)-\frac{c_{1}\left(\alpha, \gamma_{1}\right)(x+\eta)^{3} C}{B_{1}^{6}}\right\} \\
& \times\left(1-\frac{c_{2}\left(\alpha, \gamma_{1}\right)(x+\eta) C}{B_{1}^{4} \Psi(3 / 2)}\right) .
\end{aligned}
$$

Согласно условию (123), при $\gamma<\frac{1}{14^{2}}$

$$
\frac{C x}{\sqrt{\gamma} B^{4}}<\sqrt{\gamma}<\frac{1}{2 \sqrt{14}}
$$

Отсюда $L / \sqrt{\gamma}<\frac{1}{3 \sqrt{14}}$, поскольку $x>3 B / 2$. Следовательно,

$$
\frac{\eta}{B}<1,16, \quad \frac{\eta^{2}}{B^{2}}<1,16 \frac{\eta}{B}<\frac{14,98 C}{\sqrt{\gamma} B^{3}} .
$$

В силу (133) и (125)

$$
\begin{gathered}
\frac{x \eta+\eta^{2}}{B_{1}^{2}}<63,76 \frac{C x}{\sqrt{\gamma} B^{4}}<27,89 \frac{C x^{3}}{\sqrt{\gamma} B^{6}} \\
x+\eta<x+1,16 B<1,774 x .
\end{gathered}
$$

Из (132) в силу (125), (134), (135) вытекает

$$
\begin{aligned}
f(x-q)> & \left(1-\Phi\left(\frac{x}{B_{1}}\right)\right) \exp \left\{-\left(\frac{36,07}{\sqrt{\gamma}}+18,85 c_{1}\left(\alpha, \gamma_{1}\right)\right) \frac{C x^{3}}{B^{6}}\right\} \\
& \times\left(1-\frac{4 c_{2}\left(\alpha, \gamma_{1}\right) C x}{\Psi(1) B^{4}}\right) .
\end{aligned}
$$

Из (124), (125) и леммы 16 следует

$$
\begin{aligned}
1-\Phi\left(\frac{x}{B_{1}}\right) & \geqslant\left(1-\Phi\left(\frac{x}{B}\right)\right) \exp \left\{-\frac{B_{2}^{2} x^{2}}{B_{1}^{2} B\left(B_{1}+B_{2}\right) \Psi(3 / 2)}\right\} \\
& >\left(1-\Phi\left(\frac{x}{B}\right)\right) \exp \left\{-\frac{3 \sqrt{3} C x^{3}}{\sqrt{2 \gamma} B^{6} \Psi(3 / 2)}\right\}
\end{aligned}
$$


если $3 B / 2<x<\gamma B^{4} / C, \gamma<\frac{1}{14^{2}}$. Комбинируя оценки (126), (128), (136), (137), мы заключаем, что при $3 B / 2<x<\gamma B^{4} / C, \gamma<\frac{1}{14^{2}}$

$$
\mathbf{P}\{S>x\}>\left(1-\Phi\left(\frac{x}{B}\right)\right) \exp \left\{-\frac{c_{1}^{\prime}(\alpha, \gamma) C x^{3}}{B^{4}}\right\}\left(1-\frac{c_{2}^{\prime}(\alpha, \gamma) C x}{B^{4}}\right),
$$

где

$$
\begin{aligned}
& c_{1}^{\prime}(\alpha, \gamma)=18,85 c_{1}(\alpha, 3,5 \gamma)+\frac{3,68}{\sqrt{\gamma} \Psi(3 / 2)}+\frac{36,07}{\sqrt{\gamma}} \\
& c_{2}^{\prime}(\alpha, \gamma)=\frac{4 c_{2}(\alpha, 3,5 \gamma)}{\Psi(1)}+\frac{0,89}{\sqrt{\gamma}}, \quad \alpha=\sqrt{\gamma}
\end{aligned}
$$

Отсюда, подставляя вместо $c_{1}(\cdot, \cdot)$ и $c_{2}(\cdot, \cdot)$ их выражения $(22)$, мы получаем после не очень сложных вычислений требуемое неравенство.

\section{СПИСОК ЛИТЕРАТУРЫ}

1. Кендалл М. Дж., Стьюарт $A$. Теория распределений. М.: Наука, 1966, 587 с.

2. Kolmogoroff A. N. Über das Gesetz des iterierten Logarithmus. - Math. Ann., 1929, B. 101 , S. 126-135.

3. Feller $W$. Generalization of a probability limit theorem of Cramer. - Trans. Amer. Math. Soc., 1943, v. 54, № 3, p. 361-372.

4. Lenart $C$. On certain theorems of Berry and a limit theorem of Feller. - Mat. casopis, 1968 , v. 18 , № 1 , p. 59-75.

5. Петров В. В. Суммы независимых случайных величин. М.: Наука, 1972, 416 с.

6. Розовский Л.В. Одно обобщение теоремы Колмогорова о законе повторного логарифма. - Теория вероятн. и ее примен., 1997, т. 42, в. 1, с. 134-143.

7. Statulevicius V.A. On large deviations. - Z. Wahrscheinlichkeitstheor. verw. Geb., 1966, B. 6, H. 2, S. 133-144.

8. Архаягельсжий $A$. Н. Нижние оценки для вероятностей больших уклонений сумм независимых случайных величин. - Теория вероятн. и ее примен., 1989, т. 34 , B. 4 , с. $625-635$.

9. Нагаев С. В. Некоторые уточнения вероятностных неравенств. - Вестник МГУ, 1996, № 6, c. 64-66.

10. Nagaev S. V. Large deviations of sums of independent random variables. - Ann. Probab., 1979, v. 7, № 5, p. 745-789.

11. Шигаяов И. С. Об уточнении верхней оценки константы в остаточном члене центральной предельной теоремы. - Проблемы устойчивости стохастических моделей. М.: ВНИИСИ, 1982, с. 109-115.

12. Золотарев $B$. M. Современная теория суммирования независимых случайных величин. М.: Наука, 1986, 415 с.

13. Нагаев $C$. В. Некоторые предельные теоремы для больших уклонений. - Теория вероятн. и ее примен., 1965, т. 10, в. 2, с. 231-254.

14. Cramer $H$. Sur un nouveau théorème-limite de la théorie des probabilités. - Actualités Sci. Indust., 1938, № 736.

15. Нагаев C. B., Ходжабагян C. С. Об оценке функции концентрации сумм независимых случайных величин. - Теория вероятн. и ее примен., 1996, т. 41, в. 3 , c. $655-665$. 